\section{In SituDelineation Etch Reveals Subtle Detail in SEM Images of Ion Milled Cross Sections Enabling In-Fab 3-D Metrology and Characterization Clive Chandler, FEI Company}

Control of layer thickness is critically important in the manufacture of semiconductor devices. Cross-sectioning exposes device structures for direct examination but conventional sample preparation procedures are difficult, time consuming, and grossly destructive. Cross sections created by focused ion beam (FIB) milling are easier, faster, and less destructive but have not offered the clear layer delineation provided by etching in the conventional sample preparation process. A new gas etch capability (Delineation Etch ${ }^{T M}$ from FEI Company) offers results that are equivalent to conventional wet-etch preparations in a fraction of the time from a single, automated system in the fab without destroying the wafer. The new etch process also has application in milling high-aspect-ratio holes to create contacts to buried metal layers, and in deprocessing devices to reveal silicon and polysilicon structures.

Enhanced by the new delineating etch capability, FIB/SEM cross sections also offer specific benefits for new materials and processes. Copper, for instance, is notoriously difficult to polish mechanically. FIB cross sections offer clean delineation of copper structures, without smearing, for precise copper measurements. The new gas-etch does not corrode copper after processing is completed, as do other etching alternatives. Many new processes, such as dual damascene, require precise registration of structures in multiple layers and tight control of structure thickness and depth. These parameters are difficult to evaluate with conventional top-down metrology, but FIB/SEM cross sections readily expose them for direct measurement. Finally, the move to $300 \mathrm{~mm}$ wafers will increase the cost of scrapping wafers to make measurements. FIB/SEM measurements preserve the wafer intact for continued processing.

\section{Cross sections provide critical three-dimensional} information.

In order to maintain high yields as critical dimensions shrink and device structures become increasingly complex, process engineers must have high-resolution, threedimensional information. SEM (scanning electron microscope) images of device cross sections can provide the needed infor-

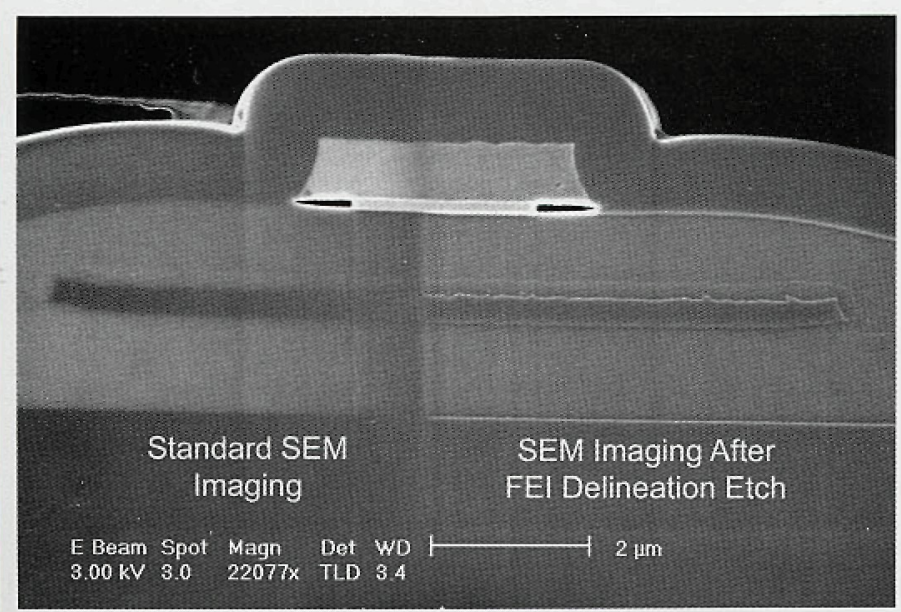

Figure 1 - The right half of this image field as been enhanced with the delineating gas-etch. Note the appearance of subtle details that are obscured or invisible in the left half. mation but conventional sample preparation techniques destroy the wafer as it is manually cleaved, polished, and etched in a laboratory outside the fab. The process requires a skilled technician and typically takes a day or more to complete.

\section{FIB cross sections are an alternative to manual sample prepa- ration.}

In recent years FIB cross-sectioning has offered an attractive alternative to the manual sample preparation process. This technique uses the ion beam to mill cross sections at a designated location in an intact wafer. It offers a number of advantages: the preservation of the wafer, preparation times on the order of one hour, and a reduction in the special skills required of the operator. Combined FIB/SEM systems (such as FEl's DualBeam ${ }^{\mathrm{TM}}$ 865) permit both cross-sectioning and high-resolution imaging in a single fab compatible system. However, prior to the introduction of the new gas-etch capability, FIB cross sections have not shown the same level layer delineation available from manual preparations.

\section{High-resolution SEM imaging is enhanced by surface topography.}

For high-resolution imaging, SEMs use the secondary electron (SE) signal. Secondary electrons are very low energy electrons inelastically scattered from sample atoms by the high-energy electrons of the SEM beam. Because of their low energy, they can escape only from a very shallow region just below the sample surface. Most SEs originate very close to the intersection of the beam with the sample surface, giving the SE signal very good spatial resolution.

Contrast in an SE image results primarily from the signal's exquisite sensitivity to local topography. The number of secondary electrons that escape from the sample and reach the detector depends strongly on the local angle between the surface and the beam and on the direction of the local slope with respect to the detector. These both change from point to point in the presence of topographic relief, generating strong image contrast. This characteristic of the secondary electron signal also makes image interpretation very intuitive. An SE image looks like a visual image of the sample surface viewed from the direction of the beam and illuminated from the direction of the detector. Surfaces are brighter or darker depending on the direction they face relative to the detector, protruding edges are highlighted and recessed edges shadowed.

\section{Etching creates delineating surface topography.}

The principal benefit of gas-etch over wet-etch is its ability to

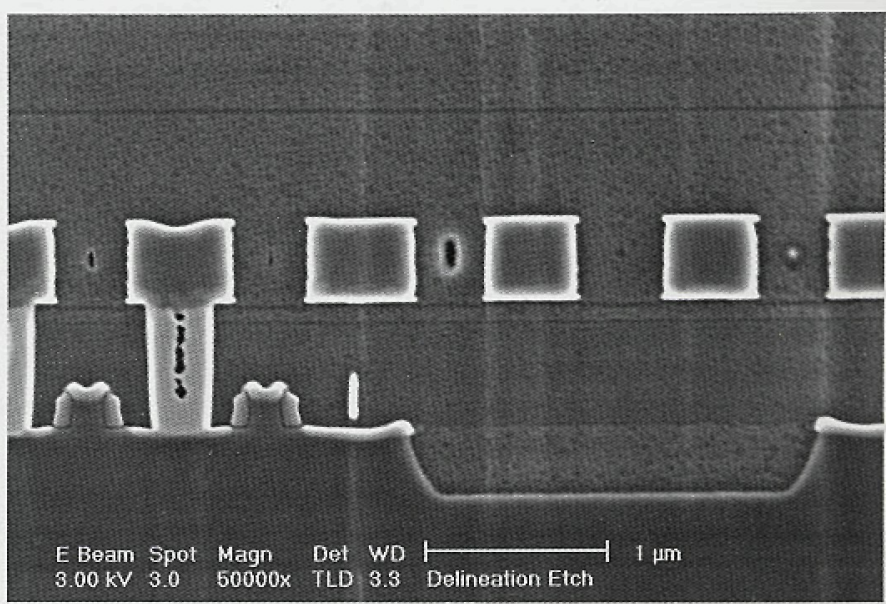

Figure 2 - The delineating etch is particularly useful in defining the interfaces between various insulator layers. 
work in the sample chamber of the DualBeam system. In fact, it requires the presence of the ion beam in order to work (Figure 1). The etching gas, as supplied, is inactive. Only when energetic ions from the beam decompose the precursor gas do the decomposition products react with the sample. The precursor gas is inherently safe for the operator and non-corrosive to the gas injection system. A gas injector introduces the gas into the sample chamber in very small amounts through a needle located close to the sample surface, thus providing a locally high concentration. The gas-etch preferentially attacks insulating materials such as oxides and does not attack silicon (crystalline or polycrystalline).

\section{Delineating etch gas chemistry works in the DualBeam} sample chamber.

The principal benefit of gas-etch over wet-etch is its ability to work in the sample chamber of the DualBeam system. In fact, it requires the presence of the ion beam in order to work (Figure 1). The etching gas, as supplied, is inactive. Only when energetic ions from the beam decompose the precursor gas do the decomposition products react with the sample. The precursor gas is inherently safe for the operator and non-corrosive to the gas injection system. A gas injector introduces the gas into the sample chamber in very small amounts through a needle located close to the sample surface, thus providing a locally high concentration. The gas-etch preferentially attacks insulating materials such as oxides and does not attack silicon (crystalline or polycrystalline).

Delineating gas-etch brings three-dimensional FIB/SEM measurement and characterization into the fab.

With FIB-based gas-etch engineers can fully utilize the powerful navigation and automation capabilities of the FIB/SEM system. One of the difficulties of manual sample preparation has always been locating the defect or structure targeted for observation. The process was often hit-or-miss and required considerable skill from the technician to cleave the wafer close to the target, then polish the cleaved surface just enough to reveal the target without destroying it. Moving the preparation process into the FIB/ SEM system eliminates most of the time, tedium, and skill required to locate the defect and reduces the chances of destroying the target while trying to find it. The system can navigate automatically to the target based on defect coordinates from an inspection tool, CAD data, electrical test data or recognized references from the circuit pattern. Once at the target location the operator can control the cross-sectioning process automatically or

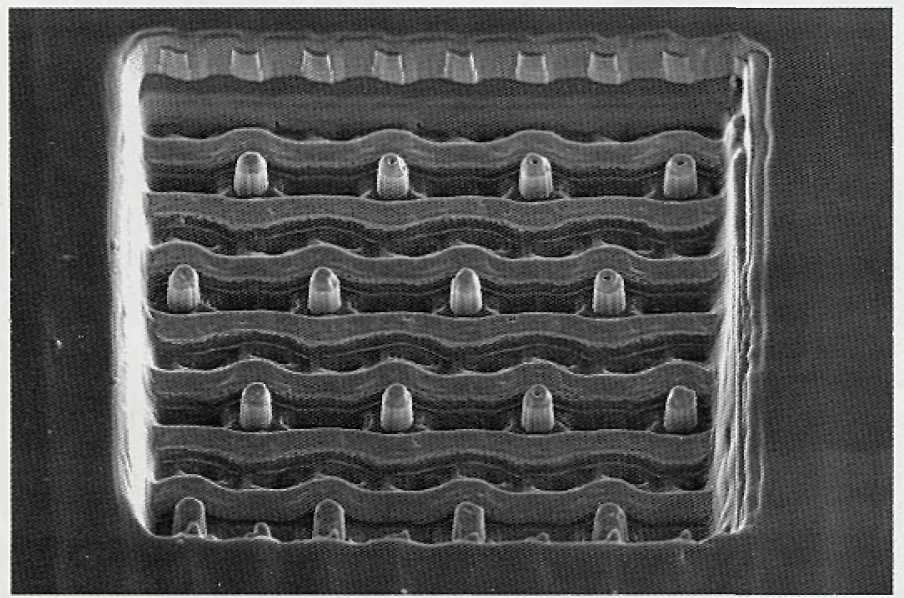

Figure 3 - Since the delineating etch gas does not attack silicon, it can be used to deprocess circuits down to the single crystal substrate and to reveal polysilicon structures. The image shows a DRAM deprocessed with the delineating etch gas. interactively, taking periodic SEM snapshots of the milling process to confirm progress and terminate when the target is fully revealed. Current generation automation offers the prospect of selecting multiple targets, milling and etching cross sections, acquiring images, and extracting critical dimensions or analytical data, all without operator intervention.

\section{Applications \\ Oxide Layer Delineation}

Figure 2 shows a device cross section prepared using the delineating gas-etch. Notice the clear demarcation of layer boundaries.

\section{Deprocessing}

Failure analysis and construction analysis applications sometimes require the selective removal of certain materials to reveal the large-scale structure of an integrated circuit. Although other etching gases, such as $\mathrm{XeF2}$, can be used with the FIB for this purpose, they have the disadvantage of aggressively attacking silicon structures as well, both polysilicon lines and the single crystal substrate. The delineating-etch gas does not attack silicon, even when activated by the beam, leaving theses structures intact for easy visualization and analysis. Figure 3 shows a DRAM circuit deprocessed to reveal the polysilicon structures.

\section{Milling High-Aspect Holes}

One of the early successes of FIB technology was its use to "rewire" integrated circuits during the development cycle to test modifications without having to generate a new mask set and process new silicon. The ion beam can cut existing metal lines and, using specially designed gas chemistries, deposit new conducting or insulating material. As circuits have become more complex with many metal layers, milling contact holes to subsurface features has become more difficult. Not only are the features deeper below the surface, but the clearances between intervening features have become smaller. The deeper, narrower holes needed to reach these features require the use of beam gas

\section{Continued on following page}

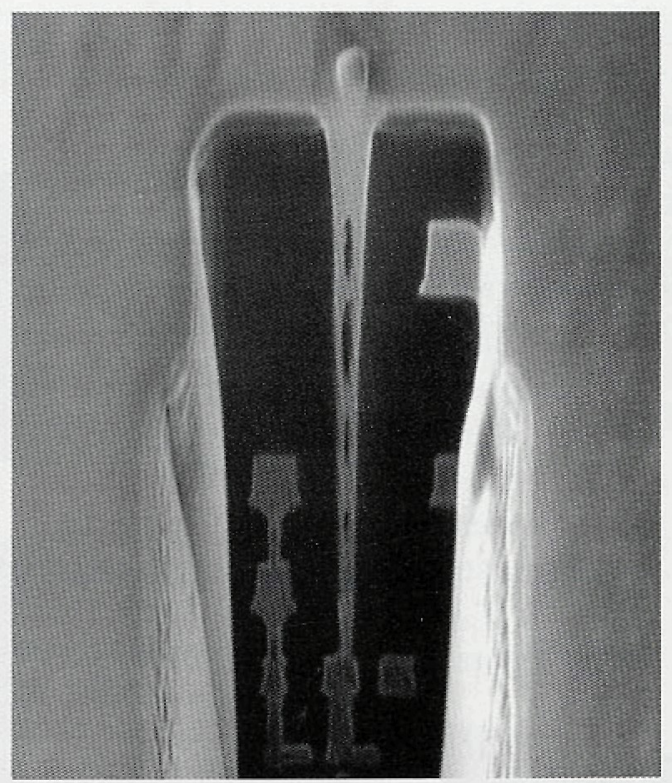

Figure 4 The delineating etch gas provides a safe alternative to $\mathrm{XeF} 2$ when milling high-aspect-ratio holes to access buried structures for circuit modification during design development. This image shows a hole 9 micrometers deep and $0.33 \mathrm{mi}-$ crometers wide at half depth, an aspect ratio of $27: 1$. 
In Situ Delineation Etch Reveals Subtle Detail in SEM Images of lon Milled Cross Sections Enabling In-Fab 3-D Metrology and Characterization

Continued from preceding page.

chemistries to prevent the redeposition of sputtered material within the hole. Although XeF2 serves well in this application, the new delineating gas offers a safer alternative for milling high-aspect holes. Figure 4 shows a contact hole milled using the delineating-etch gas.

\section{Conclusion}

FIB/SEM cross sectioning with delineating gas-etch achieves results equivalent to laboratory based sample preparation, allowing process engineers to obtain critical threedimensional measurements and perform detailed defect characterization in the fab. The technique combines crosssectioning, etching and imaging capabilities into a single, highly automated, fab-compatible system. It shortens the time-toanswer from days to hours, preserves the wafer intact for additional processing, and reduces the requirement for specialized operator skills, all of which result in a direct reduction of measurement costs and product losses. New automation capabilities promise to make the process faster and easier. Shrinking feature sizes, increasing three-dimensional complexity, new processes and materials, and larger wafers are all trends that will add to the value of FIB/SEM three-dimensional metrology and characterization in the fab.

\section{ELECTRON MICROSCOPIST}

Stanford Hospital and Clinics, part of UCSF Stanford Health Care, currently has an opportunity for a professional to process/section tissue specimens for research and diagnostic purposes utilizing both light and electron microscopes. You will photograph/process digital images, prepare frozen sections for evaluation, perform fluorescent immunostaining procedures, and train/assist faculty and staff in the use/interpretation of electron and light microscopy use. Requires a BA/BS. Prefer 2 years experience. Certification in electron microscopy a definite plus.

We offer an excellent compensation and benefits package. Send resume to: Attn: T. Ritter, email: Jobs@ ucsfstanford.org. FAX (650) 723-7205. (Please send scannable resumes: white paper; standard, 12 point fonts; no italics, underlining or graphics. Please email your resume in ASCII text or as an attachment document-Word 97 or higher)

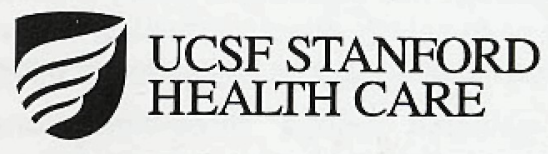

\section{www.ucsfstanford.org \\ Equal Opportunity Employer}

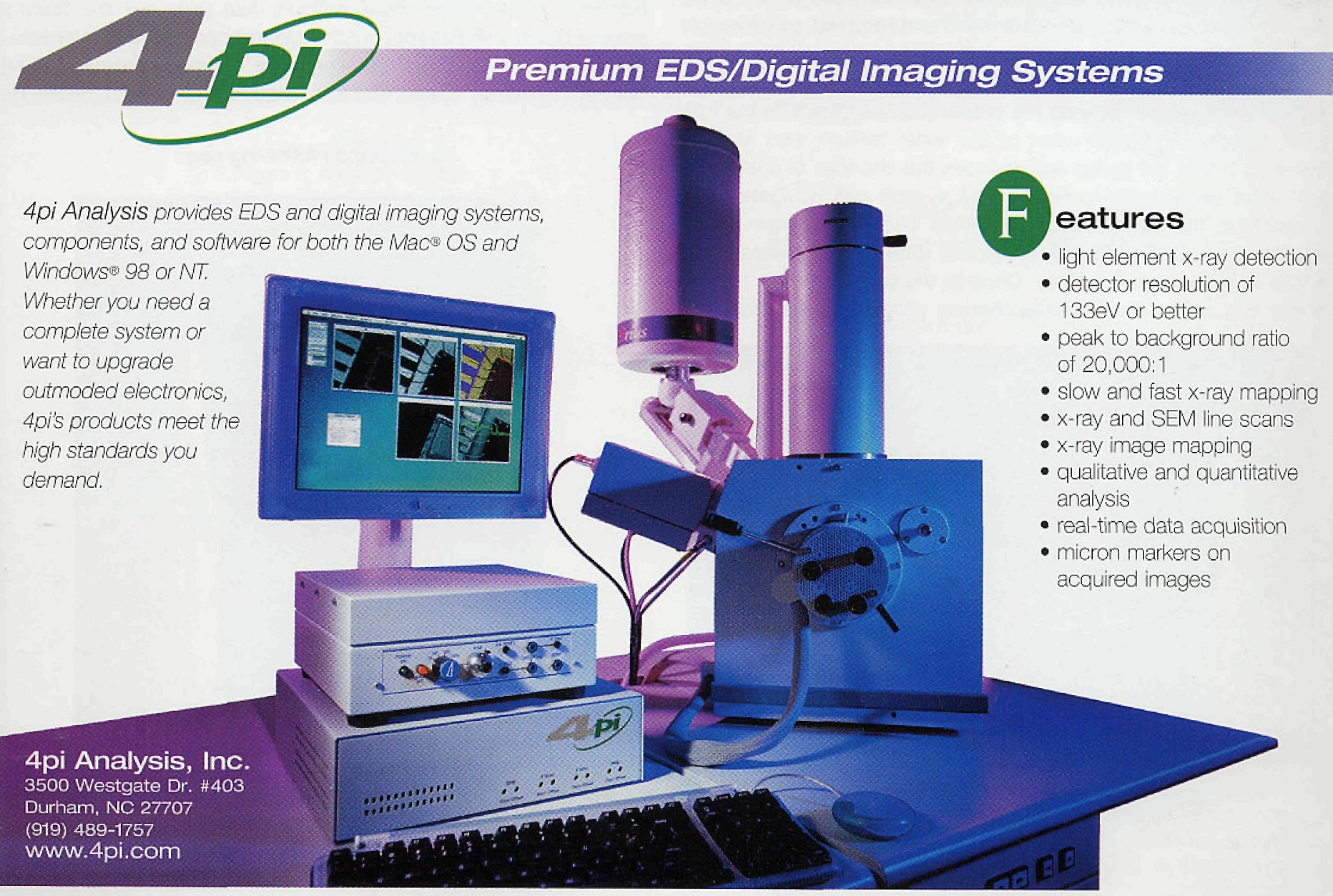



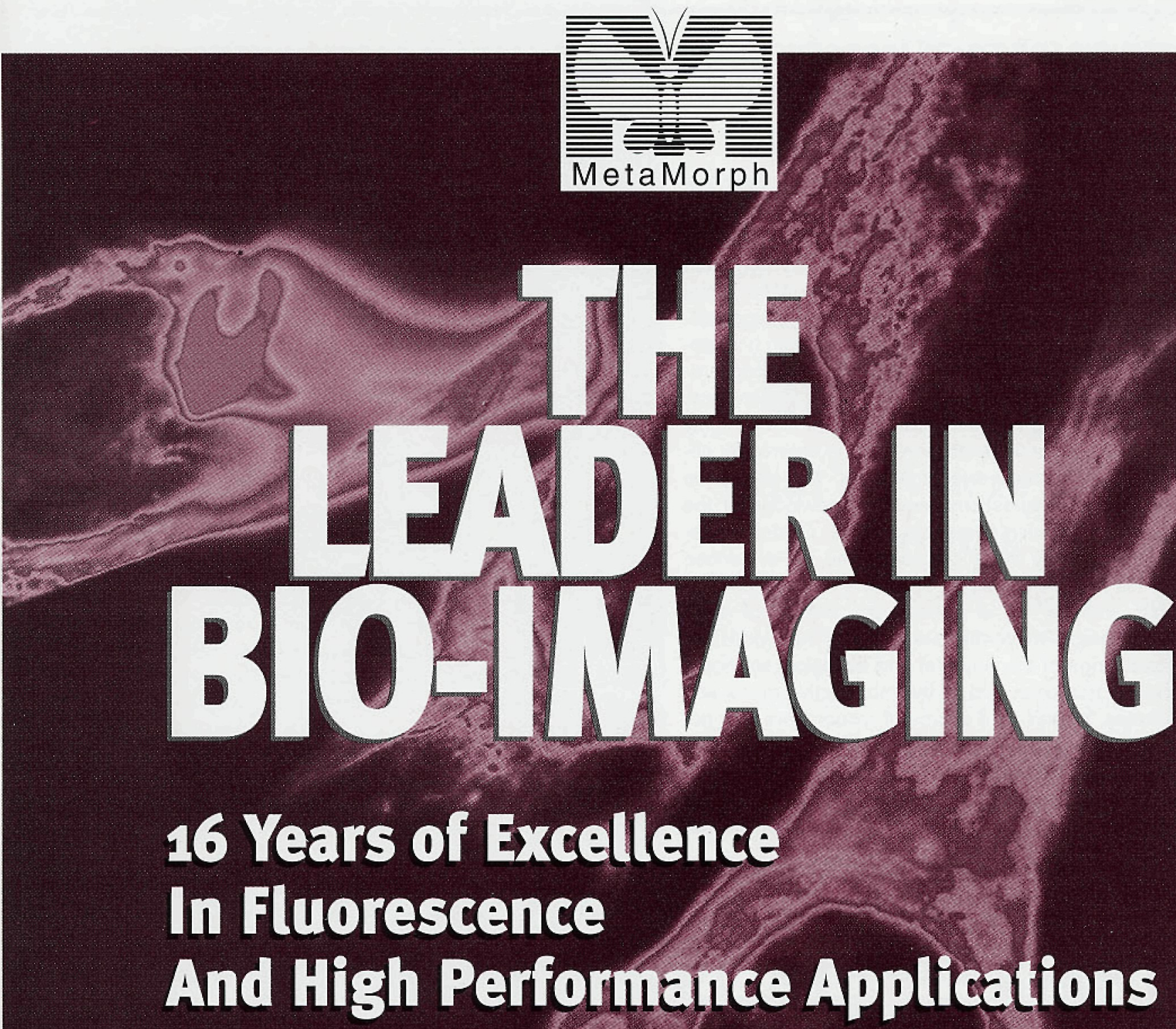

6-Dimensional Imacing GFP

Fluorescence

IMMUNOCYTOCHEMISTRY

Intracellular Calcium

Live/DeAd assays
Motion Analysis

\& Particle Trackilg

High Speed Ratlo

GLUORESCENCE

HIGH SPEED Z-SERIES

TIME LAPSE

UMARIII 